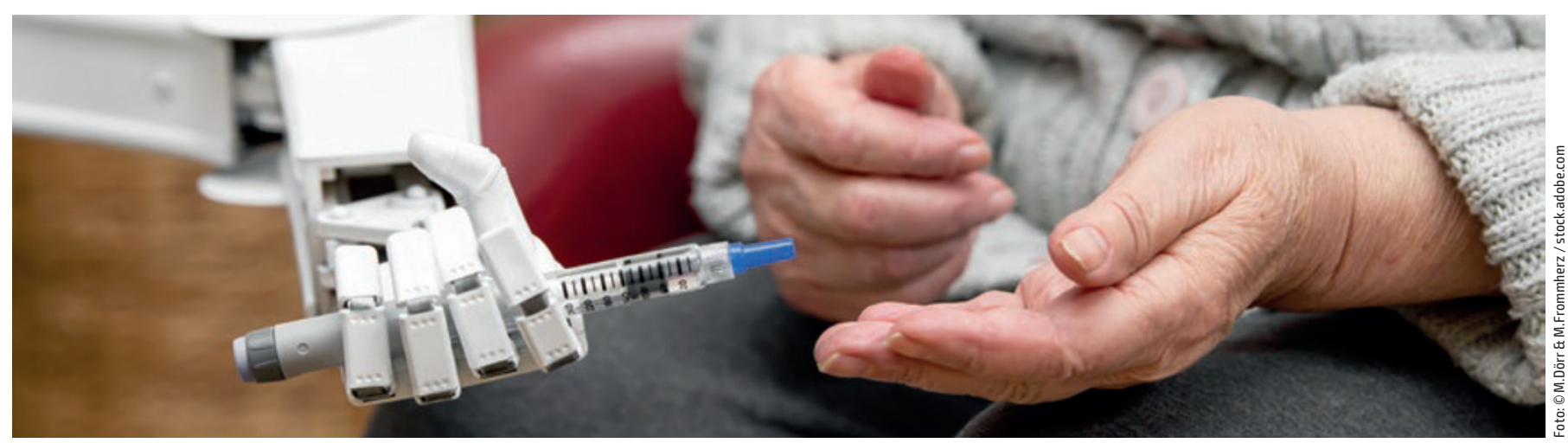

Digitale Hilfsmittel können für pflegebedürftige Personen auch kalt und unangenehm sein.

\title{
Drei Schritte für eine gute, digitale Pflege
}

Wie können digitale Technologien den Arbeitsalltag von Pflegekräften erleichtern?

Diese Frage stellt sich nicht erst seit der SARS-CoV-2-Pandemie, sondern seitdem in Österreich der chronische Personalmangel und prekäre Beschäftigungsverhältnisse zu dem sogenannten

Pflegenotstand führten. Wenn Digitalisierung für die Erleichterung des Arbeitsalltags tatsächlich

einen Beitrag leisten soll, gilt es, die zentralen Fragen rund um die Bedingungen für eine gute Pflege in den Mittelpunkt der Debatte zu rücken. Miriam Fahimi

Die politischen Diskussionen um den Pflegenotstand und Pflegearbeit als systemrelevante Arbeit im Zuge der andauernden SARS-CoV-2-Pandemie sind mittlerweile weitestgehend abgeklungen. Was bleibt, sind die weiterhin prekär und überwiegend weiblichen Beschäftigten in der Pflege, für die sich bisher kaum Verbesserungen in den Beschäftigungsbedingungen eingestellt haben. Die Beschäftigungsverhältnisse sind von einem strukturellen Mangel an Fachkräften, niedriger Entlohnung sowie physischen und psychischen Belastungen geprägt.

Währenddessen boomt der Markt für unterschiedliche Pflegetechnologien, wie beispielsweise digitale Dokumentations- und Planungssysteme, Robotiktechnologien und vernetzte Monitoring- und Assistenzsysteme. Der Einsatz von digitaler Pflegetechnologie stellt den Beschäftigten unter anderem Arbeitserleichterungen in Aussicht. Digitalisierung allein ist allerdings noch kein Garant für eine gute Pflege. Drei Schritte müssen erfolgen, wenn Digitalisierung tatsächlich den Pflegealltag erleichtern soll.

\section{Schritt 1: Ziele statt Mittel der Digitalisierung identifizieren}

Um wirksame und langfristige Erleichterungen des Pflegealltags zu erreichen, muss in einem ersten Schritt das technikzentrierte Verständnis des digitalen Wandels erweitert werden. Statt den Fokus auf die Entwicklung und Verbreitung der digitalen Mittel zu setzen, gilt es, konkrete Zielsetzungen zu definieren. Eine solche Zielsetzung kann sich beispielsweise auf die Entlastung der Pflegekräfte bei körperlich anstrengenden Aufgaben beziehen. Denn Berufstätige in der Pflege sind durch schweres Heben und Tragen einem deutlich erhöhten Risiko für die Entstehung von Muskel-Skelett-Erkrankungen ausgesetzt. Rückenbeschwerden sind eine der zentralen Ursachen dafür, warum krankheitsbedingte Fehlzeiten in der Pflege besonders hoch sind.

\section{Erst unter den geeigneten Bedingungen kann sich eine Erleichterung des Arbeitsalltags entfalten."}

Erst wenn eine konkrete Zielsetzung festgelegt wird, die auf tatsächliche Erleichterungen im Alltag der Menschen in der Pflege ausgerichtet ist, sollte danach gefragt werden, welchen Beitrag digitale Technologien zu deren Erfüllung leisten können. Verfolgen wir die Zielsetzung der Verringerung physischer Belastungen weiter, kann Robotik - unter den geeigneten Voraussetzungen und Bedingungen - als eines von mehreren Mitteln definiert werden. Durch Mobilisations- und Heberobotik können pflegebedürftige Personen beispielsweise so bewegt werden, dass der Rü- 
cken der Pflegekräfte kaum belastet wird. Genauso wichtig sind aber auch analoge Mittel, wie Präventionsmaßnahmen für eine rückenschonende Arbeitsweise im Rahmen von Aus- und Weiterbildungsprogrammen zu schulen, und dass im Arbeitsalltag ausreichend Zeit und Raum vorhanden sind, um diese umzusetzen.

\section{Schritt 2: Bedürfnisorientierte Technologie gemeinsam entwickeln}

Digitale Technologien werden aufgrund ungleicher Machtverhältnisse am Arbeitsmarkt oft abgekoppelt von der Pflege entwickelt. In den seltenen Fällen, in denen es zu einer Zusammenarbeit zwischen Ingenieurs- und Pflegewissenschaften kommt, zeigen sich große Herausforderungen aufgrund unterschiedlicher Zugänge und auszuhandelnder Begriffsverständnisse. Umso wichtiger ist es, dass gesetzte Ziele, den Arbeitsalltag in der Pflege zu erleichtern, von Beginn an Eingang in den Entwicklungsprozess digitaler Technologien finden. Pflegewissenschaften müssen dabei in diesen Prozess eingebunden werden. Ebenfalls müssen die Bedürfnisse aller Akteurinnen und Akteure in der Pflege - der Beschäftigten, der pflegebedürftigen Personen und der Angehörigen - Teil des Entwicklungsprozesses sein. Deren Bedürfnisse können sich dabei voneinander unterscheiden. Im Fall der vorher beispielhaft genannten Zielsetzung der physischen Entlastung bedeutet das: Mobilisations- und Heberobotik kann zwar Pflegekräfte entlasten, sich für pflegebedürftige Personen aber auch kalt und unangenehm anfühlen.

Auch sind nicht alle Beschäftigten, pflegebedürftigen Personen und Angehörigen eine jeweils homogene Gruppe mit gleicher Meinung. Einer Studie mit US-amerikanischen Pflegekräften zufolge nimmt die jüngere Generation der Pflegekräfte eine offenere Haltung gegenüber digitalen Technologien ein als ältere Berufstätige in der Branche. Auch gesellschaftliche Geschlechterrollen spielen weiterhin eine Rolle, denn männliche Pflegekräfte haben einer quantitativen Befragung nach eine positivere Einstellung gegenüber dem Einsatz digitaler Technologien. Allerdings ist eine positive Resonanz immer nur unter der Voraussetzung festzustellen, dass mit der Digitalisierung keine Einsparungen beim Personal einhergehen.

\section{Schritt 3: Rahmenbedingungen der Digitalisierung gestalten}

Das Argument, dass technologischer Fortschritt zu einer gesellschaftlichen Aufwertung von Pflegearbeit führe, existiert seit Jahrzehnten. Bisher lässt sich eine Aufwertung empirisch nicht feststellen. Anstelle von Autonomiegewinnen für alle Berufsgruppen in der Pflege führt technologischer Fortschritt eher zu einer verstärkten Polarisierung von hoch- und niedrigqualifizierter Pflegearbeit.

Die Umsetzung von Arbeitsalltagserleichterung muss somit in einem dritten Schritt über die Digitalisierung hinausgehen. Erst unter den geeigneten Bedingungen kann sich eine Erleichterung des Arbeitsalltags auch wirklich entfalten. Dabei gilt es beispielsweise zu verhindern, dass Pflegekräften noch mehr Tätigkeiten auferlegt werden, da Rationalisierungs- und Effizienzgewinne über den vermehrten Einsatz digitaler Technologien erwartet werden. Es geht aber auch darum, dass Sorge- und Beziehungsarbeit als gesellschaftlich relevante Arbeit anerkannt und

\section{NICHT PRIMÄR TECHNISCHE ANTWORTEN}

Digitalisierungsprozesse sind nicht statisch, universell oder von vornherein festgelegt, sondern gestaltbar. Dementsprechend ist die Idee, dass Digitalisierungsprozesse von außen auf die Pflege treffen und automatisch für einen erleichterten Arbeitsalltag sorgen würden, abzulehnen. Die skizzierten Schritte zu einer guten, digitalen Pflege im Sinne aller Gruppen im Pflegebereich ziehen umfassende gesellschaftliche Fragen nach sich: Wird der Arbeitsalltag erleichtert, wenn effizient und kostensparend gepflegt werden kann? Wird der Arbeitsalltag erleichtert, wenn ausreichend Zeit für Beziehungsarbeit und eine individuelle, ganzheitliche Betreuung vorhanden ist? Und wird der Arbeitsalltag erleichtert, wenn Berufstätige in der Pflege gerecht entlohnt werden, ausreichend Zeit für Pausen haben und gesundheitsfördernd arbeiten können? Solche Fragen verlangen nicht primär nach technischen Antworten. Vielmehr gilt es in erster Linie auszuhandeln, welche Pflege wir uns als Gesellschaft - d. h. für unsere Eltern, Kinder, Freundinnen, Freunde und schließlich auch für uns selbst - wünschen.

entsprechend strukturell aufgewertet wird. Weiterhin spielen persistierende gesellschaftliche Zuschreibungen an Geschlechterrollen eine wesentliche Rolle, die Sorgearbeiten insbesondere Frauen zuweisen und als niedrigqualifizierte Arbeit abwerten. In Folge wird Pflegearbeit insbesondere im Bereich der Langzeitpflege trotz ihrer gesellschaftlichen Relevanz und des erforderlichen fachlichen Know-hows prekär entlohnt. Die Forderung der Beschäftigten in der Pflege und deren interessenpolitischer Vertretungen nach einer Verkürzung der wöchentlichen Arbeitszeit bei vollem Lohn- und Personalausgleich kann u.a. richtungsweisend für progressive Konzepte zur Verbesserung der Arbeitsbedingungen sein.

Doch nicht allein die Pflegeorganisationen sind gefordert, wenn es darum geht, die Arbeitsbedingungen und die Bezahlung der Beschäftigten zu verbessern. Die Gestaltungsmöglichkeiten und die Handlungsmacht der Pflegeorganisationen reichen nur so weit wie ihre Finanzierung durch Bund und Länder. Allerdings gehört Österreich mit Bruttoausgaben für Pflege in Höhe von 1,5 Prozent des BIP im EU-weiten Vergleich weiterhin zu den Schlusslichtern. Neben der so wichtigen Forderung nach Arbeitszeitverkürzung ist der Blick daher verstärkt auf die staatliche Ausfinanzierung der Pflege zu richten.

\section{Literatur bei der Autorin}

Dieser Textbeitrag ist unter einer Creative-Commons-Lizenz vom Typ Namensnennung - Weitergabe unter gleichen Bedingungen 4.0 International (CC BY-SA) zugänglich. Um eine Kopie dieser Lizenz einzusehen, konsultieren Sie http://creativecommons.org/licenses/by-sa/4.0/ Weitere Informationen auch hier: Open Access: Zielsetzung und Verwendung - Arbeit\&Wirtschaft Blog (awblog.at)

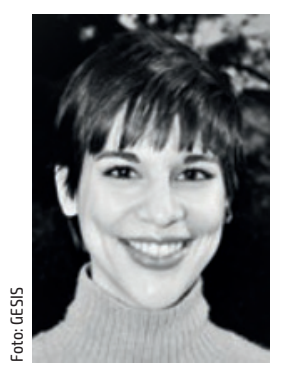

\section{MIRIAM FAHIMI, MA, BSC,} SOZIALWISSENSCHAFTLERIN

Marie Skłodowska-Curie Fellow im EU-Projekt NoBIAS - Artificial Intelligence without Bias, Wissenschaftliche Mitarbeiterin an der Alpen-Adria-Universität Klagenfurt. Für ihre Masterarbeit an der Universität Wien erhielt Fahimi den AK-Wissenschaftspreis 2020 miriam.fahimi@aau.at 